\title{
INCORPORAÇÃO DE RODAMINA B EM MEMBRANA DE POLIAMIDA PELO MÉTODO DA ADSORÇÃO
}

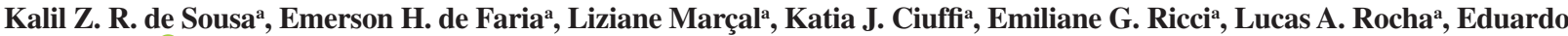 \\ J. Nassar ${ }^{a, *,(1)}$, Jorge V. L. Silva ${ }^{\text {b }}$, Marcelo F. Oliveira ${ }^{\text {b }}$ Izaque A. Maia ${ }^{\text {b }}$ \\ aUniversidade de Franca, 14404-600 Franca - SP, Brasil

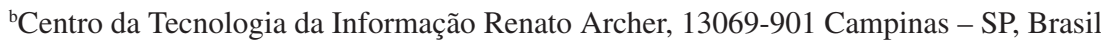

Recebido em 04/08/2020; aceito em 19/10/2020; publicado na web em 11/11/2020

\begin{abstract}
RODAMINE B INCORPORATION INTO POLYAMIDE MEMBRANE BY ADSORPTION METHOD. We have incorporated rhodamine into polyamide membranes by additive manufacture selective laser sintering technology. Incorporation into $0.5 \mathrm{~cm}^{2}$ membranes was conducted at different $\mathrm{pH}$ values and constant temperature of $25^{\circ} \mathrm{C}$. The amounts of incorporated dye were determined by a calibration curve, and analyses showed rhodamine incorporation in the order of $10^{-6} \mathrm{~mol} \mathrm{~L}^{-1}$. The absorption spectra of the solutions and membranes after rhodamine incorporation showed maximum absorption at $553 \mathrm{~nm}$ and a shoulder at $522 \mathrm{~nm}$, which indicated that a dimer was formed. Photoluminescence presented maximum emission at $575 \mathrm{~nm}$ for the sample incorporated with rhodamine in acid $\mathrm{pH}$. The membranes that were treated with acid presented shorter adsorption time and demanded $180 \mathrm{~min}$ to reach equilibrium, whereas the non-treated membrane and the membranes that were treated with base required $1440 \mathrm{~min}$ to reach equilibrium. Polyamide membrane has proven to be an excellent material for the incorporation of dyes and compounds for application as luminescent sensors.
\end{abstract}

Keywords: additive manufacture; photoluminescence; zero charge potential.

\section{INTRODUÇÃO}

Nos últimos anos, a Prototipagem Rápida (PR) conhecida também como Manufatura Aditiva (MA) e/ou Impressão 3D, vem evoluindo devido à avançada tecnologia dos equipamentos utilizada para tal, passou de uma simples metodologia de preparação de protótipos para a indústria para uma sofistica ferramenta de preparação de peças com tamanhos e formas complexas. A MA é particularmente adequada para a fabricação de praticamente qualquer forma de material ou dispositivo. ${ }^{1,2}$ As peças produzidas por essa técnica têm tido um crescimento rápido e contínuo em diferentes campos industriais, desde aplicações automotivas, aeroespaciais e biomédicas. A metodologia permite a criação de uma vasta gama de dispositivos em pouco tempo com vários tipos de materiais, polímeros e metais. A qualidade das peças impressas em $3 \mathrm{D}$ podem ter suas propriedades mecânicas comprometidas devido a sua complexidade e avaliações do comportamento desses materiais devem ser constantemente estudadas, sendo que a literatura tem relatado trabalhos neste sentido..$^{3-5}$

A utilização de polímeros como componente em vários setores industriais tem se tornado mais complexas, resultando sua aplicação como material estrutural. Os processos de fabricação dessas peças evoluíram e hoje a MA tem sido essencial para satisfazer novos requisitos. ${ }^{6}$

A Sinterização Seletiva a Laser (SLS) é uma das tecnologias aditivas e tem se destacado devido seu maior potencial de obtenção de peças com altas propriedades mecânicas, estabilidade térmica e excelente qualidade da superfície, quando comparada com outras técnicas como a Deposição por Fusão (FDM) ${ }^{5,7}$ A técnica SLS utiliza feixe de laser para fusão do polímero, a poliamida 12 (PA12) ainda é o polímero mais utilizado. ${ }^{8,9}$ As peças produzidas com PA12 podem sofrer modificações químicas utilizando processos como a metodologia sol-gel, ativando sua superfície, a qual pode acomodar diferentes íons e/ou moléculas ajustando suas propriedades para diferentes aplicações como biomateriais, sensores, liberação controlada de fármacos, entre outras. ${ }^{10-17}$

\footnotetext{
*e-mail: eduardo.nassar@unifran.edu.br
}

A Rodamina $\mathrm{B}(\mathrm{RB})$ é um corante catiônico pertencente à família dos compostos orgânicos xantenos (estrutura básica) sendo sua fórmula molecular $\left(\mathrm{C}_{28} \mathrm{H}_{31} \mathrm{~N}_{2} \mathrm{O}_{3} \mathrm{Cl}\right)$, altamente luminescente com emissão no intervalo de comprimento de onda de $580-600 \mathrm{~nm}$, devido a sua propriedade espectroscópica tem sido utilizado como sensores para matéria orgânica, gases, ânions e cátions. ${ }^{18,19}$ Aplicações estão relacionadas a sensores de alta sensibilidade para íons $\mathrm{Cu}^{2+}{ }^{20-}$ ${ }^{26}$ Essas características são atribuídas às propriedades fotofísicas, fazendo dessa molécula um poderoso quimiosensor fluorescente que pode sofrer mudanças em seus comprimentos de onda de absorção e emissão, apresenta alto coeficiente de absorção e alto rendimento quântico de fluorescência. ${ }^{27}$

Outra aplicação que tem chamado atenção para a RB são em lasers aleatórios (randômicos), isso se deve a fácil e econômica produção, ${ }^{28} \mathrm{com}$ aplicação em diagnósticos médicos. ${ }^{29-31}$ Esse tipo de laser é diferente dos convencionais, o corante é incorporado em um meio altamente dispersante que pode amplificar a luz, sistemas coloidais, ${ }^{32}$ pós semicondutores, ${ }^{33}$ vidros fotônicos, ${ }^{34}$ pontos quânticos coloidais, ${ }^{35}$ e matrizes como a silica. ${ }^{36,37}$

Inúmeros estudos na literatura têm apontado em direção da obtenção por sensores luminescentes com alta sensibilidade em baixas concentrações tem atraído a utilização de corantes orgânicos como a rodamina $\mathrm{B}$, em associação com sistemas hospedeiros com propriedades físico-químicas que possam acomodar esses sensores. Neste trabalho, membranas de PA 12 com espessura de $200 \mu \mathrm{m}$ foram produzidas por manufatura aditiva utilizando, para isso, a técnica de sinterização seletiva a laser. $\mathrm{O}$ corante $\mathrm{RB}$ foi incorporado às membranas em diferentes $\mathrm{pH}$ e caracterizadas por espectroscopias eletrônica e vibracional, fotoluminescência e análise térmica.

\section{PARTE EXPERIMENTAL}

\section{Preparo da membrana de poliamida 12}

As membranas de poliamida 12 (PA12) obtidas por manufatura aditiva (MA) e usando a técnica de sinterização seletiva a laser (SLS), foram tratadas, conforme descrito na literatura por nosso grupo. ${ }^{10-12} \mathrm{As}$ 
membranas foram cortadas em pedaços de dimensões $0,5 \mathrm{~cm} \times 0,5 \mathrm{~cm}$ ou $0,25 \mathrm{~cm}^{2}$ (Figura 1) e posteriormente lavadas para que não houvesse resíduos de pó da poliamida após o processo. Em um béquer, as membranas nas dimensões acima foram colocadas em $30 \mathrm{~mL}$ de água destilada, deixando em agitação magnética por $60 \mathrm{~min}$. Em seguida, as membranas foram retiradas e secas à temperatura ambiente. A PA foi adquirida pelo CTI da 3D Systems, DuraForm PA \& GF Materials Typical Properties for SLS Systems, EUA, densidade $0,59 \mathrm{~g} \mathrm{~cm}^{-3} \mathrm{e}$ tamanho de partícula $58 \mu \mathrm{m}$.

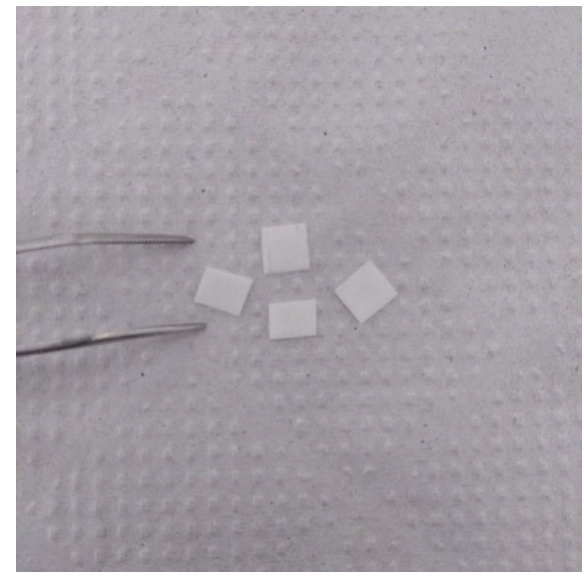

Figura 1. Membranas utilizadas no experimento

\section{Pré-tratamento ácido e básico}

As membranas foram pré-tratadas com solução $1,0 \mathrm{~mol} \mathrm{~L}^{-1}$ de ácido acético e hidróxido de amônio sob agitação magnética por $24 \mathrm{~h}$. As mesmas foram lavadas com água destilada em banho de ultrassom e secas à temperatura ambiente por mais $24 \mathrm{~h}$. Esse tratamento foi realizado a fim de ativar a superfície da membrana e estudar o melhor tratamento para adsorção da RB.

\section{Preparo das soluções aquosas de Rodamina B para obtenção da curva de calibração}

Preparou-se uma solução aquosa estoque de RB na concentração $1,0 \times 10^{-3} \mathrm{~mol} \mathrm{~L}^{-1}$ em um balão volumétrico de $50 \mathrm{~mL}$. A partir desta solução e de cálculos de diluição, preparou-se cinco outras soluções, de concentrações: $1,0 \times 10^{-6}, 2,5 \times 10^{-6}, 5,0 \times 10^{-6}, 7,5 \times 10^{-6}$,

(a) Curva de Calibração - Soluções Padrões de Rodamina

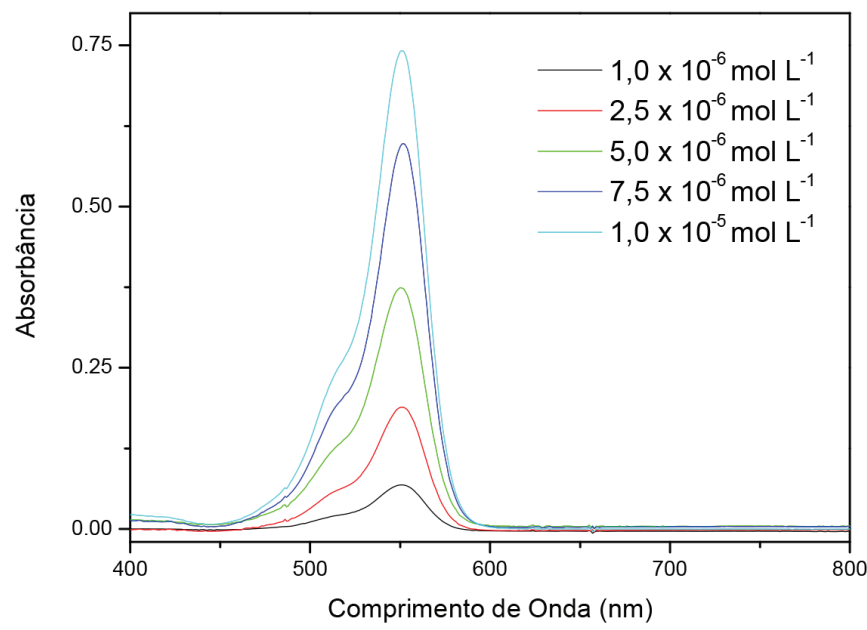

$1,0 \times 10^{-5} \mathrm{~mol} \mathrm{~L}^{-1}$ em balões volumétricos de $25 \mathrm{~mL}$. As medidas de absorbância das soluções foram realizadas no espectrofotômetro HP 8453. Por meios dos espectros das soluções padrão de RB com máximo de absorção em $550 \mathrm{~nm}$ (Figura 2a), obteve-se a curva de calibração (Figura 2b) utilizada no trabalho, que apresentou um $\mathrm{R}^{2}=0,99857$, apresentando confiabilidade na equação da reta gerada $(y=-0,0016+77192,30769 x)$ nos dados para sua utilização na quantificação das concentrações do corante remanescentes nos sobrenadantes após a adsorção.

\section{Preparo de soluções para obtenção do ponto de carga zero (PCZ) da membrana de PA12}

O potencial de carga zero para as membranas de PA12 foram realizadas utilizando as soluções de $\mathrm{NaCl}, \mathrm{HCl}$ e $\mathrm{NaOH}$ 0,1 mol L-1. Para o processo de obtenção do PCZ utilizou um reator com suporte para dez tubos de ensaio. $15 \mathrm{~mL}$ da solução de cloreto de sódio $(\mathrm{NaCl})$ $0,1 \mathrm{~mol} \mathrm{~L}^{-1}$ foram transferidas para 10 tubos de ensaio, o $\mathrm{pH}$ das soluções foram ajustados em 2, 3, 4, 5, 6, 7, 8, 9, 10 e 11 utilizando $\mathrm{HCl}$ e $\mathrm{NaOH} 0,1 \mathrm{~mol} \mathrm{~L}^{-1}$ com auxílio de um pHmetro, conforme descrito na literatura. ${ }^{38}$

A cada tubo de ensaio foram adicionadas uma membrana $\left(0,25 \mathrm{~cm}^{2}\right.$, Figura 1$)$ e uma barra magnética, os tubos foram colocados no suporte do reator e acoplado ao banho termostático, para manter a temperatura constante em $25{ }^{\circ} \mathrm{C}$. Os tubos foram mantidos em agitação magnética (1300 rpm) durante $24 \mathrm{~h}$.

\section{Influência do pH na adsorção da RB nas membranas de PA12}

Em um meio de adsorção, o pH pode afetar o grau de ionização das impurezas, assim como a carga superficial do adsorvente. Além disso, a carga superficial do adsorvente influencia na cinética da adsorção e na concentração de equilíbrio da solução. ${ }^{39}$

$\mathrm{O}$ pH de uma solução é um parâmetro de controle importante no processo de adsorção ${ }^{40}$ e esse parâmetro afeta a carga superficial dos adsorventes, bem como afeta o grau de ionização de diferentes corantes. Os íons hidrogênio e hidroxilas são fortemente adsorvidos e, por isso, a adsorção de outros íons é afetada pelo pH da solução. A variação no valor do $\mathrm{pH}$ afeta o processo de adsorção pela dissociação de grupos funcionais nos sítios ativos da superfície do adsorvente.

A adsorção da RB nas membranas de PA12 em função do pH foi realizada utilizando-se uma solução de concentração $1,0.10^{-5} \mathrm{~mol} \mathrm{~L}^{-1}$ em água. Em $15 \mathrm{~mL}$ da solução de RB foi adicionada a membrana de

(b)

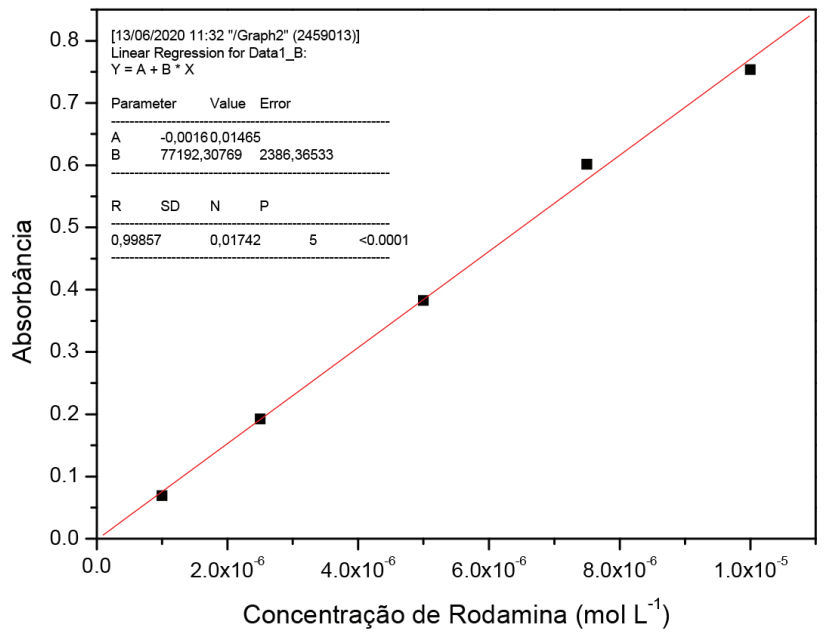

Figura 2. Espectros de absorção das soluções de Rodamina B em diferentes concentraçães (a) e curva de calibração $\left(R^{2}=0,99857\right)(b)$ 
poliamida e tendo seus $\mathrm{pH}$ ajustados em 2, 3, 4, 5, 6, 7, 8, 9, 10 e 11, a Figura 3 mostra a foto do sistema utilizado. Os pHs foram medidos antes e após a incorporação em período de $24 \mathrm{~h}$. As membranas foram secas à temperatura ambiente após o tempo estipulado.

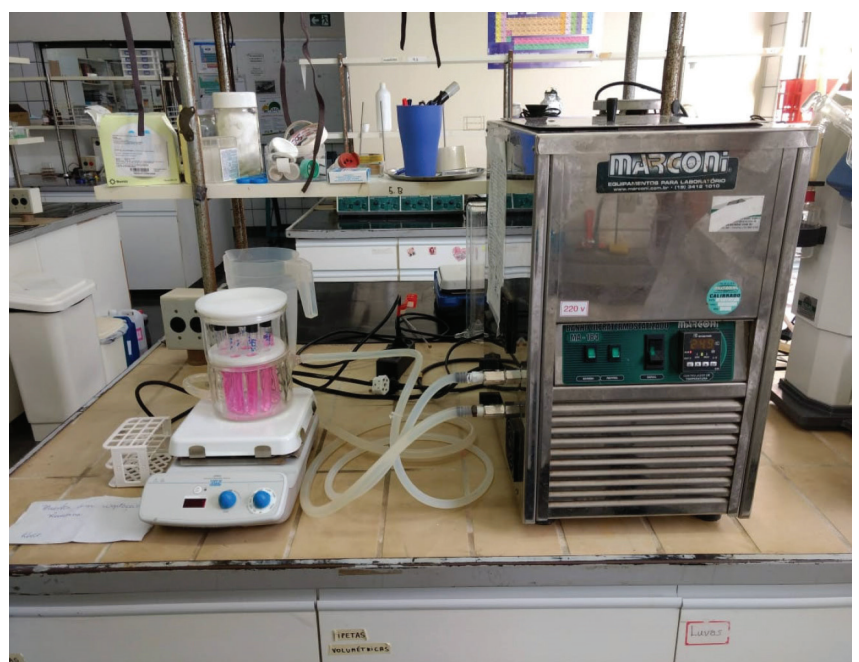

Figura 3. Sistema utilizado na incorporação da RB nas membranas de PA12 à temperatura controla de $25^{\circ} \mathrm{C}$

\section{Caracterizações}

A espectroscopia vibracional na região do infravermelho (FTIR) foram registradas a partir de 4000 à $600 \mathrm{~cm}^{-1}$ no modo ATR em espectrômetro Perkin-Elmer Frontier. A espectroscopia eletrônica de sólidos foi realizada em um Ocean Optic por reflectância difusa com uma sonda e lâmpada pulsada de Xe PX-2 e detector QE65000 e os espectros de absorção das soluções em um Hewlett-Packard 8453 diode array. Os espectros de emissão da RB foram realizados em um Horiba Jobin Yvon Fluorolog-3 equipado com duplo monocromador e fotomutiplicadora Hammatsu R 928. As análises térmicas utilizaram um TA Instruments-SDT Q600-Simultaneous DTA-TGA, razão de aquecimento de $10{ }^{\circ} \mathrm{C} \mathrm{min}^{-1}$.

\section{RESULTADOS E DISCUSSÃO}

O ensaio de potencial de carga zero é uma importante informação para entender o mecanismo da adsorção, uma vez que é possível verificar o comportamento das cargas superficiais das amostras dos adsorventes, facilitando a compressão dos processos de agregação dispersão na operação de adsorção.

A determinação da carga superficial em sólidos é um importante parâmetro para processos adsortivos, o PCZ pode ser definido como o pH em que a superfície apresenta carga zero. $\mathrm{O}$ valor do $\mathrm{pH}$ em que a adsorção de íons depende da concentração dos íons $\mathrm{H}^{+}$e $\mathrm{OH}^{-}$, permitindo prever e estimar a ionização dos grupos funcionais e como quais espécies esses grupos poderão interagir. ${ }^{41}$

A solução de cloreto de sódio apresentava pH inicial de 7,26, o qual foi ajustado à 2, 3, 4, 5, 6, 7, 8, 9, 10 e 11 e colocada em contato com as membranas de poliamida. Após 24 h de agitação, as membranas foram retiradas e secas. Os pHs das soluções foram medidos antes e após contato com as membranas de poliamida. A Figura 4 apresenta o gráfico dos $\mathrm{pHs}$ finais em função dos $\mathrm{pHs}$ iniciais das soluções de cloreto de sódio.

A superfície pode atingir carga positiva e/ ou negativa, dependente do $\mathrm{pH}$, promovendo a protonação ou desprotonação dos grupos funcionais superficiais. ${ }^{42}$ A Figura 4 apresenta o $\mathrm{pH}$ final em função do $\mathrm{pH}$ inicial (curva preta). Observam-se algumas alterações entre o

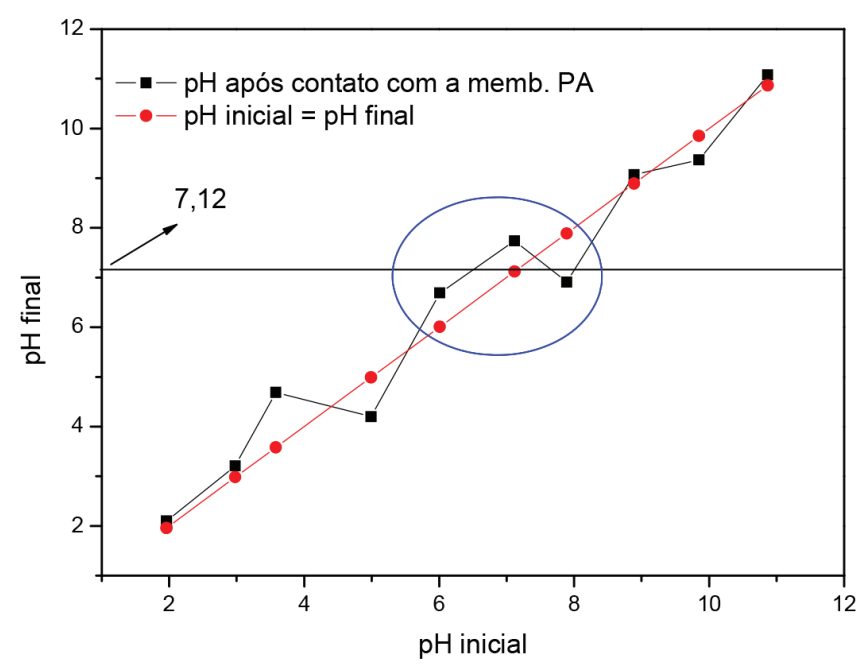

Figura 4. $p H$ final em função do $p H$ inicial, experimental (preto) e teórico (vermelho)

pH inicial e final, porém, o PCZ da superfície encontra-se ente 6 e 8 , valor médio de 7,12. Ao traçar a curva $\mathrm{pH}$ inicial $=\mathrm{pH}$ final (curva vermelha), levando em consideração a não variação do $\mathrm{pH}$, teria PCZ igual $\mathrm{pH} 7$, muito próximo do observado experimentalmente, e nesse ponto a concentração de $\mathrm{H}^{+}$se iguala a de $\mathrm{OH}^{-}$. Assim, o gráfico indica que abaixo de pH 6 ocorre a protonação do grupo funcional amida e sua carga é positiva, enquanto acima de $\mathrm{pH} 8$ ocorre um aumento de cargas negativas na superfície da membrana de poliamida. ${ }^{43}$

De modo que a adsorção do cátion é favorecida quando o $\mathrm{pH}$ da solução é maior que o PCZ, enquanto que a adsorção de ânions é favorecida quando o $\mathrm{pH}$ é menor que o PCZ.

As membranas foram analisadas por espectroscopia vibracional na região do infravermelho, a Figura 5 apresenta os espectros de FTIR das membranas após tratadas nos diferentes pHs e secas. A Figura 5 apresenta os espectros das membranas tratadas com pH ácido (2), neutro (7) e básico (11), além da membrana sem tratamento.

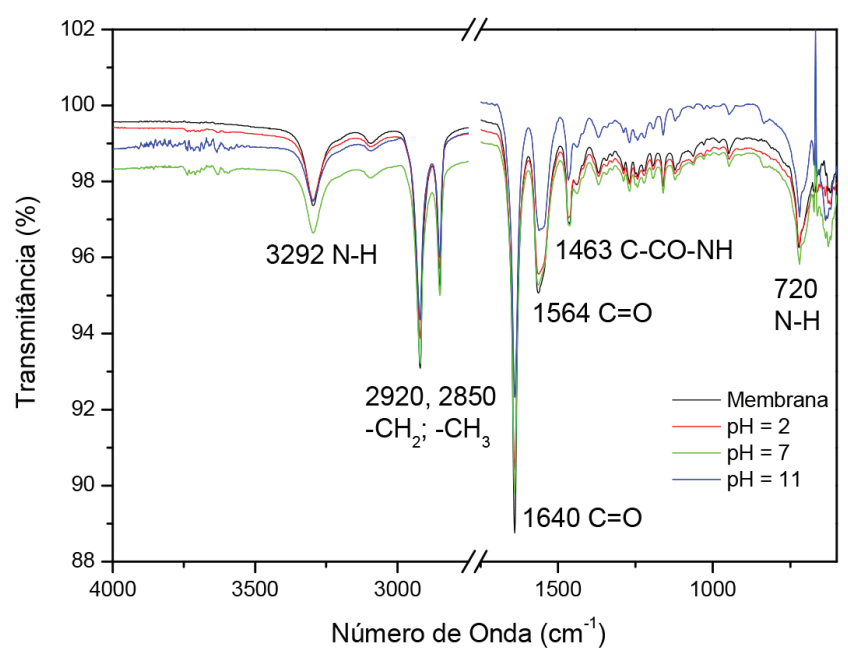

Figura 5. Espectros de infravermelho das membranas PA12 sem tratamento e após 24 h nas soluções de $\mathrm{pH}=2,7$ e 11

A Figura 5 apresenta os modos vibracionais característico da PA12 ${ }^{10-14}$ e não foram observadas alterações e nem deslocamentos nos picos, o que pode ser um indicativo de que as cargas superficiais não foram suficientes, o $\mathrm{pH}$ ao qual foram submetidas as membranas não afetou a estrutura superficial da membrana de PA12. Picos de absorção em 3292 e 720 cm$^{-1}$ foram atribuídos a vibrações N-H, 2920 
e $2850 \mathrm{~cm}^{-1} \mathrm{CH}_{2}$ e $\mathrm{CH}_{3}, 1640$ e $1564 \mathrm{~cm}^{-1}$ ao grupo $\mathrm{C}=\mathrm{O}$ e $1463 \mathrm{~cm}^{-1}$ ao grupo amida (C-CO-NH).

A Tabela 1 apresenta as atribuições das bandas de absorção no infravermelho referentes à membrana de PA12 tratada nos diferentes pHs.

Tabela 1. Atribuições das principais vibrações no infravermelho de poliamida

\begin{tabular}{cc}
\hline Picos & Atribuições \\
\hline $1463 \mathrm{~cm}^{-1}$ & $v_{\mathrm{C}-\mathrm{N}}$ (amida) \\
$1553 \mathrm{~cm}^{-1}$ & $\delta_{\mathrm{NH}}$ (amida II) \\
$1640 \mathrm{~cm}^{-1}$ & $v_{\mathrm{C}=\mathrm{O}}$ (amida I) \\
2850 e $2920 \mathrm{~cm}^{-1}$ & $v_{\mathrm{C}-\mathrm{H}}$ \\
$3292{\mathrm{e} 720 \mathrm{~cm}^{-1}}^{-1}$ & $v_{\mathrm{N}-\mathrm{H}}$ \\
\hline
\end{tabular}

A RB apresenta-se em diferentes formas, dependente do $\mathrm{pH}$ e do solvente, forma catiônica em pH menor que 4, zwiteriônica em $\mathrm{pH}$ maior que 4 e em solventes apróticos lactônica. ${ }^{44} \mathrm{~A} \mathrm{RB}$ foi incorporada à membrana de PA12 em diferentes $\mathrm{pH}$ para verificação de qual a forma é mais favorável para a incorporação, foram utilizados os mesmos pHs do experimento anterior para determinação do PCZ da membrana. A solução aquosa de RB 1,0 $\times 10^{-5} \mathrm{~mol} \mathrm{~L}^{-1}$ apresentou $\mathrm{pH}$ inicial de 8,34 , os $\mathrm{pHs}$ foram ajustados conforme descrito na seção experimental.

As membranas de PA12, ao serem colocadas em solução aquosa, apresentam densidade menor e pouca interação superficial com a água. Após o tempo de reação, as membranas apresentaram um aumento de densidade e maior molhabilidade, e visualmente a membrana foi ao fundo da solução. A Figura 6 mostra as fotos das membranas após incorporação da RB nos diferentes pHs e observa-se a coloração característica da RB, sendo esse um indicativo da incorporação da RB na PA12.

As membranas foram caracterizadas através de análise térmica (TGA e sua derivada), espectroscopia vibracional, espectros de absorção de sólidos e determinação da quantidade incorporada através das medidas de absorção das soluções antes e após incorporação.

Os máximos de absorção da RB mostraram-se dependente do $\mathrm{pH}$ da solução utilizada para a incorporação. As amostras com $\mathrm{pH}$ ácido (2, 3 e 4) apresentou máximo de absorção em 554 nm, para as soluções com pH 5, 6 e 7 em $550 \mathrm{~nm}$ e para as soluções básicas (8, 9, 10 e 11) em $547 \mathrm{~nm}$. Grimm et al. ${ }^{45}$ demonstraram em seu trabalho que as propriedades fotofísicas e químicas da rodamina podem ser modificadas através de substituições químicas, proporcionando absorção e emissão em diferentes comprimentos de onda. O deslocamento do máximo de absorção observado neste trabalho pode estar relacionado à região de interação entre a RB e a PA12. Na forma catiônica, a RB apresenta em sua molécula carga positiva superficial, podendo interagir com grupos doadores de elétrons da superfície da

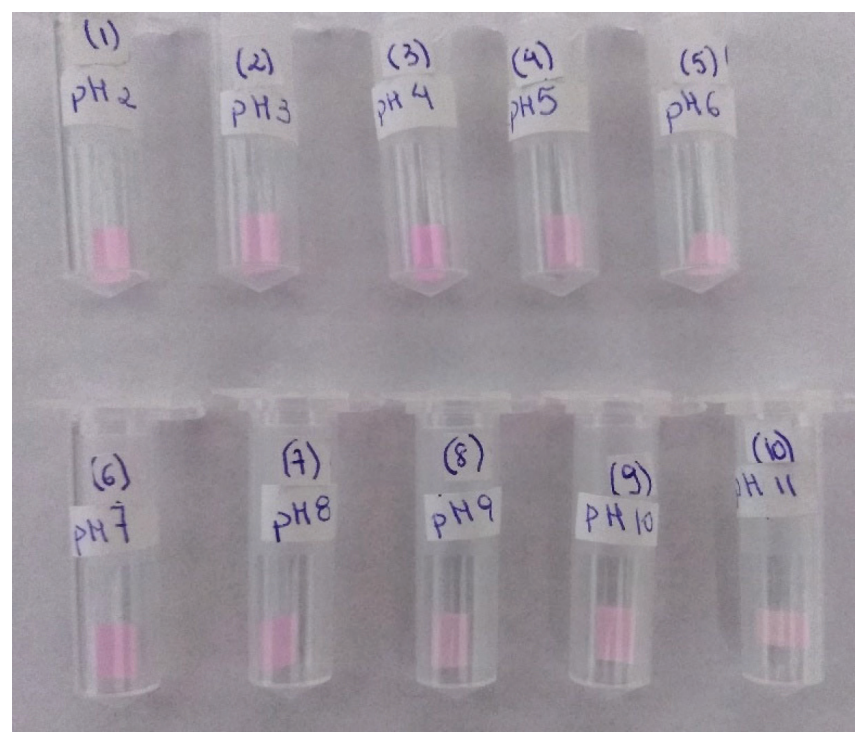

Figura 6. Fotos das membranas após a incorporação da RB em diferentes $\mathrm{pHs}$

membrana, o que ocorre para $\mathrm{pH}<4$. A forma zwiteriônica (ocorre em $\mathrm{pH}>4$ ), as moléculas apresentam cargas positivas no grupo amina e negativas na carbonila, ${ }^{44}$ podendo assim, interagir com a membrana em regiões diferentes, promovendo deslocamento do máximo de absorção.

Segundo Lacerda et al., ${ }^{46}$ a diminuição da adsorção quando o $\mathrm{pH}$ da solução foi superior a 8 pode ser devido à formação de íons hidratados de rodamina $\mathrm{B}$, que leva a uma estrutura molecular maior (dimer), que dificultam a entrada do corante nos poros do adsorvente.

A RB em solução aquosa tende a formar agregados, isso é, dímeros, trímeros, ou mesmo polimerizarem entre outros, dependente do solvente e concentração, tendo influência nas propriedades espectroscópicas. $\mathrm{O}$ monômero se apresenta no espectro de absorção com máximo ao redor de $550 \mathrm{~nm}$, enquanto o dímero em $520 \mathrm{~nm}$. ${ }^{47-49}$ A Figura 7 apresenta os espectros de absorção da RB na membrana. A figura foi dividida em três partes, dependendo do $\mathrm{pH}$ da solução utilizada para a incorporação.

A Figura 7a apresenta um máximo em 554 nm e um ombro em $520 \mathrm{~nm}$ para a RB incorporada utilizando $\mathrm{pH} 2,3$ e 4, o ombro é um indicativo que a RB se encontra na forma de dímero. Nas Figuras $7 b$ e 7c o ombro em aproximadamente $520 \mathrm{~nm}$ vai desaparecendo com o aumento do $\mathrm{pH}$, indicando a incorporação na forma monomérica do corante. A banda de absorção ao redor de $554 \mathrm{~nm}$ é atribuída a transição $\pi \rightarrow \pi^{*}$ dos orbitais HOMO para o LUMO do sistema conjugado da RB. ${ }^{50}$

As Figuras $8 \mathrm{a}$ e $8 \mathrm{~b}$ apresentam as análises térmicas, curva TGA e DTG para a membrana de PA12 antes da incorporação e após a incorporação da RB.
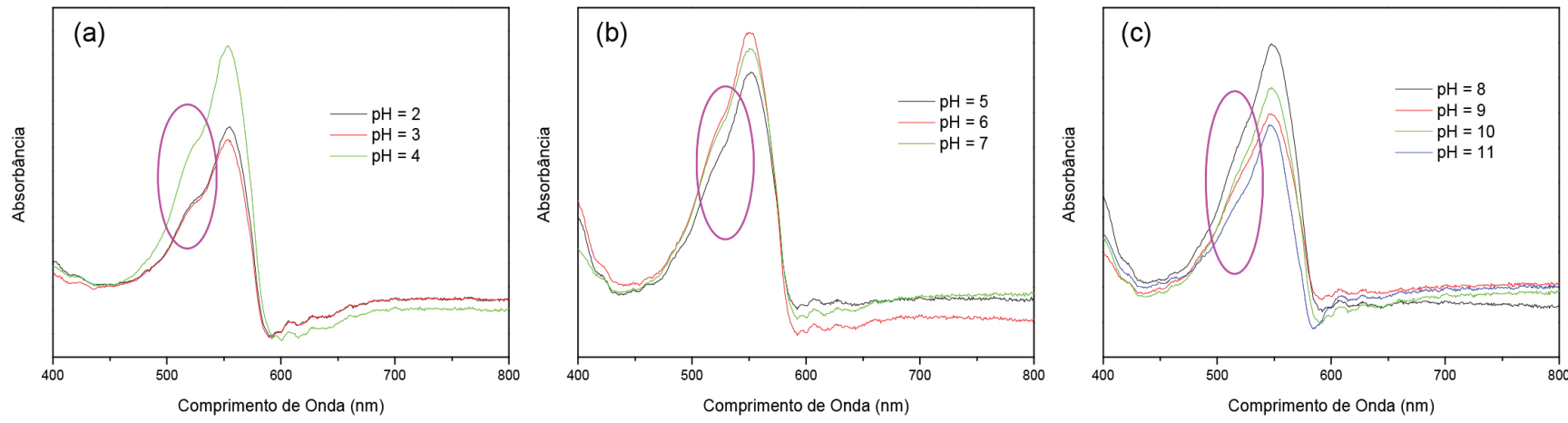

Figura 7. Espectros de absorção da RB incorporada à membrana de PA12, a) $p H=2,3$ e 4; b) $p H=5,6$ e 7 e c) $p H=8,9,10$ e 11 


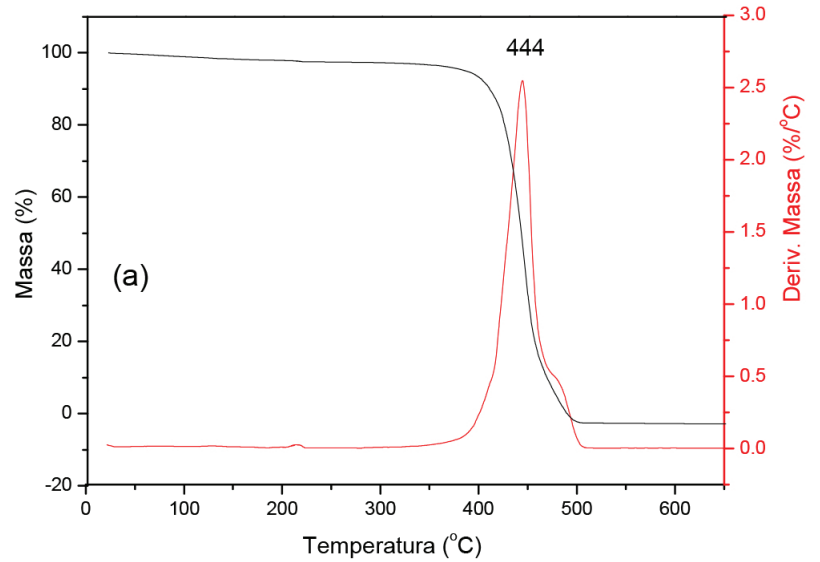

Figura 8. Curvas TGA e DTG das membranas sem (a) com RB (b)

A análise térmica apresentou pouca diferença entre a membrana pura e a membrana após incorporação da RB, sendo que nesse caso foi utilizada a amostra que foi incorporada com o $\mathrm{pH}=7$. As temperaturas máximas de decomposição são próximas, $444{ }^{\circ} \mathrm{C}$ para a PA 12 e $440{ }^{\circ} \mathrm{C}$ para a PA12 com RB. A diferença não é significativa por ter sido utilizada uma razão de aquecimento de $10{ }^{\circ} \mathrm{C} \mathrm{min}{ }^{-1}$. O intervalo de decomposição medido na DTG mostra um aumento com relação a PA12 pura, $367-503{ }^{\circ} \mathrm{C}$ para $347-503{ }^{\circ} \mathrm{C}$, aumento que pode estar relacionado à decomposição da $\mathrm{RB}$, que deve ocorrer próximo à decomposição da PA.

Os espectros de FTIR para as membranas após incorporação da RB não apresentaram vibrações características da RB, por apresentar modos vibracionais nas mesmas regiões PA12 e por estar em concentração na ordem de $10^{-6} \mathrm{~mol} \mathrm{~L}^{-1}$, como veremos a seguir na determinação da quantidade adsorvida na membrana. A Figura 9 apresenta a concentração de RB incorporada nas membranas em função do pH da solução.

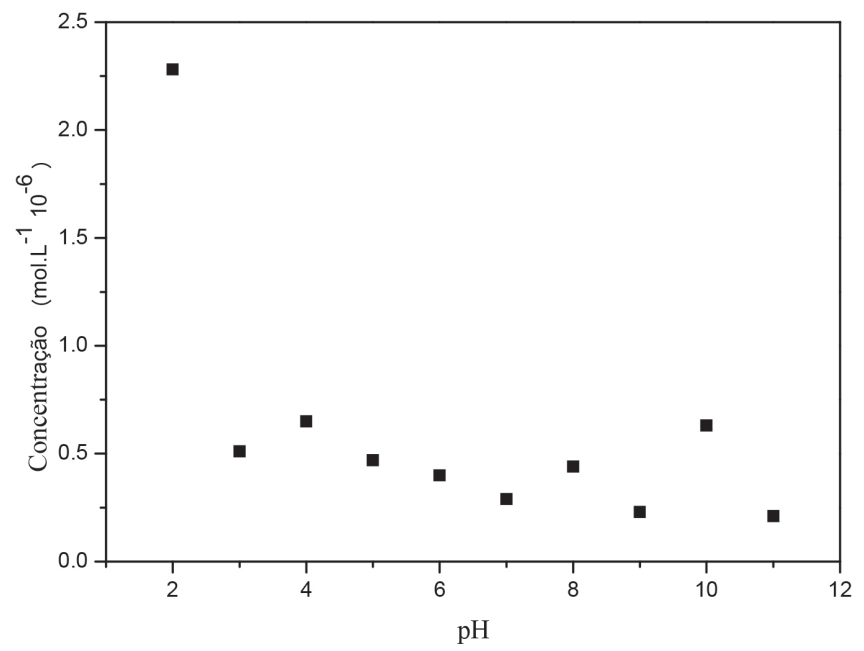

Figura 9. Quantidade de RB incorporada à membrana nos diferentes $p H$

A incorporação realizada em $\mathrm{pH}=2$ apresentou 4 vezes mais incorporação de RB na membrana, nesse pH a RB encontra-se na forma de dímero e catiônica, forma essa que prevaleceu após incorporação, como pode se observado nos espectros de absorção das membranas sólidas (Figura 7).

As membranas de PA12 foram tratadas com ácido e base, ativando a sua superfície com cargas positivas e negativas, respectivamente, conforme discutido anteriormente. Foi realizado o estudo do tempo de adsorção da RB nas membranas de PA12 em

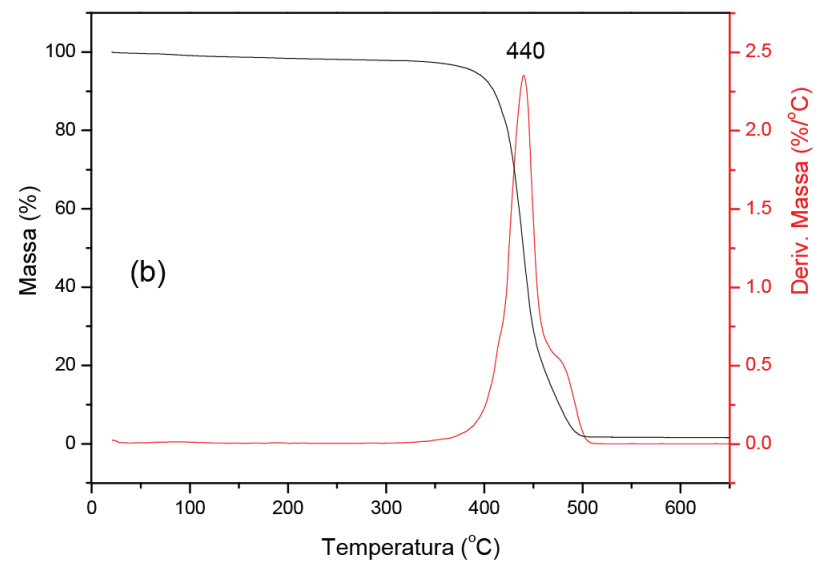

função do tratamento a que foram submetidas e em uma membrana se tratamento. A Figura 10 apresenta o decréscimo da concentração em função do tempo.

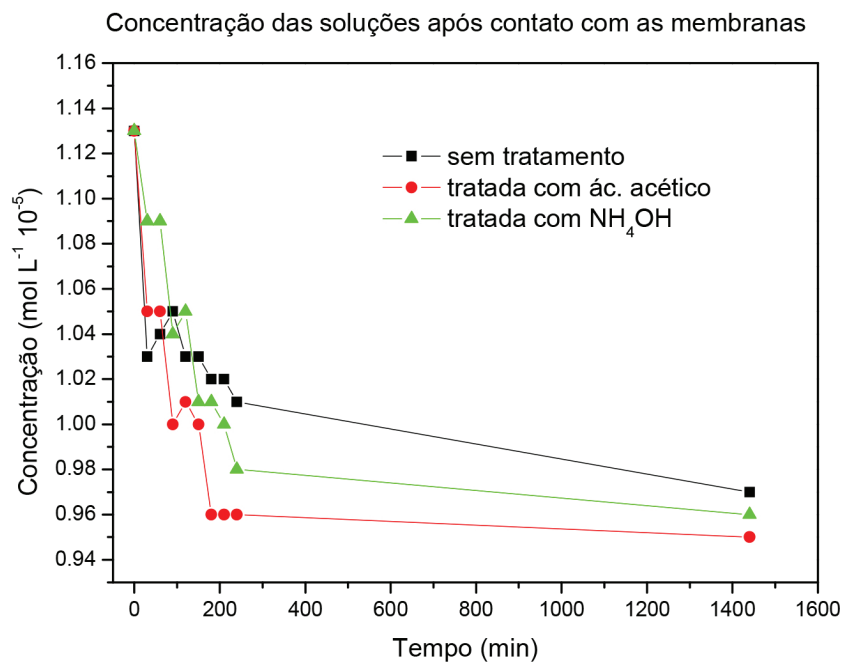

Figura 10. Concentração da solução em função do tempo de contato com a membrana de PA12

A Figura 10 mostra e confirma a tendência de maior adsorção da membrana após ser tratada com ácido, isso é, deixando a superfície com carga positiva. A membrana sem nenhum prétratamento apresenta a menor adsorção. A membrana tratada com ácido necessita de menos de 200 min para estabilizar, enquanto a membrana sem tratamento e a tratada com base precisaram de mais de $200 \mathrm{~min}$.

A alta sensibilidade da RB e outros corantes e suas propriedades espectroscópicas, permite aplicações como corantes em células, tecidos e animais. ${ }^{45}$ Assim, medidas de fotoluminescência foram realizadas, a Figura 11 apresenta espectro de emissão da RB em solução na concentração de $1,0 \times 10^{-5} \mathrm{~mol} \mathrm{~L}^{-1}$, excitado em $350 \mathrm{~nm}$, comprimento de onda obtido a partir do espectro de excitação da solução de RB.

O espectro de emissão da solução é semelhante ao observado por da Silva et al. ${ }^{51} \mathrm{O}$ máximo da banda de emissão da RB pode variar dependente do substituinte utilizado. Grimm et al. ${ }^{45}$ utilizando vários ligantes, observaram uma variação no comprimento de onda de emissão de 546 à 664 nm, emissão que vai da região verde do espectro eletromagnético até o vermelho passando pelo laranja. $\mathrm{O}$ máximo observado na solução de RB desse trabalho em solução aquosa foi em $577 \mathrm{~nm}$. 
Espectro de emissão $\lambda_{\text {exc }}=350 \mathrm{~nm}$ solução $1,010^{-5} \mathrm{~mol} \mathrm{~L}^{-1}$

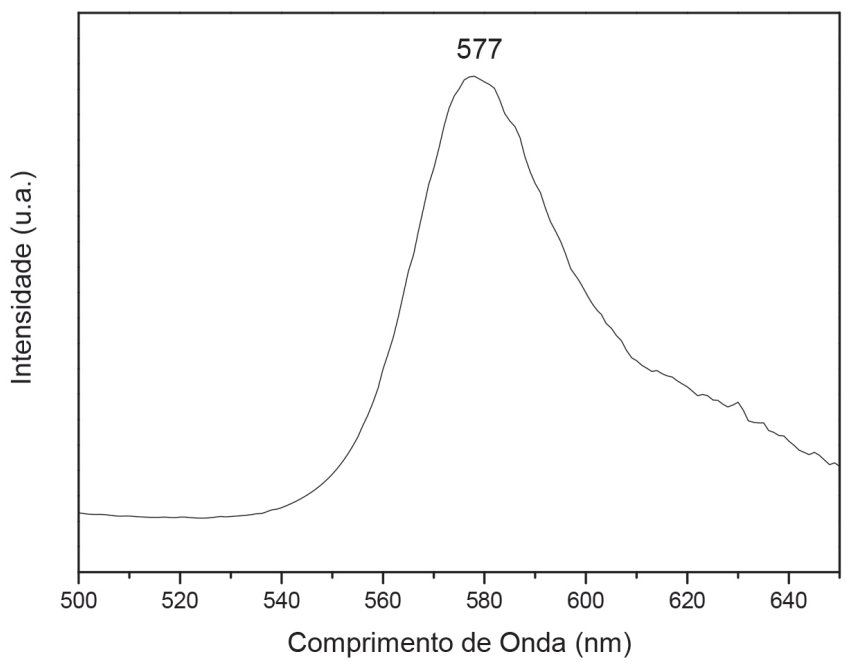

Figura 11. Espectro de emissão da solução de RB 1,0 $\times 10^{-5} \mathrm{~mol} \mathrm{~L}^{-1}, \lambda_{\text {exc }}=$ $350 \mathrm{~nm}$

Os espectros de emissão da RB incorporada a membrana PA12 tratada com ácido e base e sem tratamentos prévios são mostrados na Figura 12.

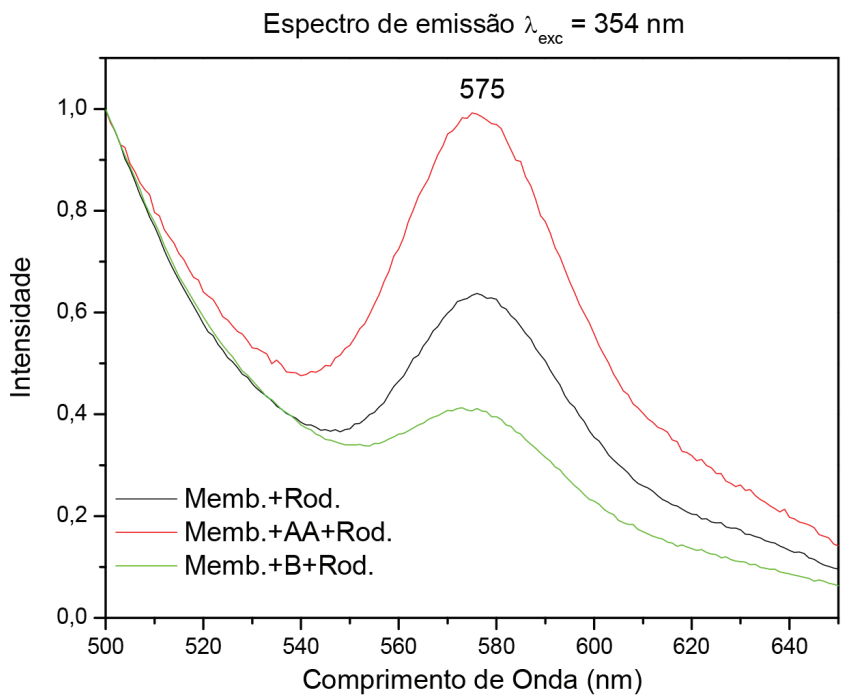

Figura 12. Espectros de emissão da RB incorporada na membrana PA12 tratada com ácido e base e sem tratamento

A Figura 12 mostra máximo de emissão em 575 nm, característica da RB, sugerindo a maior emissão para a RB incorporada na membrana após tratamento ácido, fato que pode ser relacionado à melhor interação do RB com a carga superficial da PA12 positiva.

\section{CONCLUSÕES}

A incorporação de corantes com propriedades espectroscopicas altamente luminescentes em vários tipos de matrizes abrem importante campo de aplicações como sensores e lasers aleatórios. A manufatura aditiva se mostra como uma importante tecnologia para a preparação de peças poliméricas com diferentes formas e tamanho, podendo produzir sistemas para diversas aplicações. Neste trabalho, membranas de poliamida foram preparadas pela tecnologia de sinterização seletiva a laser e tratadas e utilizadas como matriz para suportar o corante rodamina B. A incorporação proporcionou a conservação das propriedades fotofísicas do corante em baixas concentrações, abrindo caminho para várias aplicações. $\mathrm{O}$ pH 2 foi o que se mostrou melhor na incorporação da rodamina $\mathrm{B}$. O potencial de carga zero para a membrana ocorreu ao redor de $\mathrm{pH} 7$ e mostrou que os tratamentos químicos aos quais foram submetidas as membranas não as afetaram estruturalmente, mostrando a resistência química da poliamida.

A MA apresenta-se hoje como uma tecnologia para a produção de peças poliméricas com formas, tamanho e complexidades variadas, as quais podem ser utilizadas em diversos ramos da ciência. A incorporação de moléculas luminescentes, como a RB, pode atuar como sensores e/ou aplicações em lasers randômicos. Este trabalho mostrou que a incorporação da RB em concentração na ordem de $10^{-6} \mathrm{~mol} \mathrm{~L}^{-1}$ em membrana de PA, sem modificação de suas propriedades fotofísicas, abre caminho para as aplicações pretendidas e supracitadas.

\section{AGRADECIMENTOS}

Esse estudo foi parcialmente financiado pela Coordenação de Aperfeiçoamento de Pessoal de Nível Superior - Brasil (CAPES) Finance Code 001, Fundação de Amparo à Pesquisa do Estado de São (FAPESP, processos 2015/20298-0, L.A.R, e 2019/02641-0, E.J.N) e Conselho Nacional de Desenvolvimento Científico e Tecnológico (CNPq, processos 302702/2018-0, L.A.R., e 302668/2017-9, E.J.N.).

\section{REFERÊNCIAS}

1. Volpato, N. Em Prototipagem Rápida: Tecnologias e Aplicações, Edgard Blücher: São Paulo, 2007.

2. Du, Y.; Chen, J.; Meng, Q.; Dou, Y.; Xu, J.; Shen, S. Z.; Vacuum 2020, 178, 109384.

3. Santonocito, D.; Procedia Structural Integrity 2020, 25, 355.

4. Roszak, D. S. R.; Sagradov, I.; Sparr, H.; Ziegenhorn, M.; Kupsch, A.; Léonard, F.; Müller, B. R.; Bruno, G.; Arch. Mech. 2019, 71, 507.

5. Wudy, K.; Drummer, D.; Addit. Manuf. 2019, 25, 1.

6. Schob, D.; Sagradov, I.; Roszak, R.; Sparr, H.; Franke, R.; Ziegenhorn, M.; Kupsch, A.; Léonard, F.; Müller, B. R.; Bruno, G.; Eng. Fract. Mech. 2020, 229, 106841 .

7. Schmid, M.; Selektives Lasersintern (SLS) mit Kunststoffen Technologie, Prozesse und Werkstoffe, Carl Hanser Verlag: München, 2015.

8. https://wohlersassociates.com/press71.html, acessada em outubro 2020.

9. Lanzl, L.; Wudy, K.; Drummer, D.; Polym. Test.2020, 83, 106313.

10. de Souza, M. L.; Moscardini, S. B.; de Faria, E. H.; Ciuffi, K. J.; Rocha, L. A.; Nassar, E. J.; Silva, J. V. L.; Oliveira, M. F.; Maia, I. A.; Quim. Nova 2018, 41, 519 .

11. de Souza, É. A.; Rocha, L. A.; de Faria, E. H.; Ciuffi, K. J.; Nassar, E. J.; Silva, J. V. L.; Oliveira, M. F.; Maia, I. A.; J. Inorg. Biochem. 2018, 180, 171.

12. de Souza, É. A.; de Campos, B. M.; Rocha, L. A.; de Faria, E. H.; Ciuffi, K. J.; Nassar, E. J.; Silva, J. V. L.; Oliveira, M. F.; Maia, I. A.; Quim. Nova 2016, 39, 1044.

13. de Campos, B. M.; Nassar, E. J.; Calefi, P. S.; Ciuffi, K. J.; Silva, J. V. L.; Oliveira, M. F.; Maia, I. A.; J. Therm. Anal. Calorim. 2014, 115, 1029.

14. Bandeira, L. C.; Ciuffi, K. J.; Calefi, P. S.; Nassar, E. J.; Silva, J. V. L.; Oliveira, M. F.; Maia, I. A.; Salvado, I. M.; Fernandes, M. H. V.; J. Braz. Chem. Soc. 2012, 23, 810.

15. Nassar, E. J.; Bandeira, L. C.; de Campos, B. M.; Calefi, P. S.; Ciuffi, K. J.; Silva, J. V. L.; Oliveira, M. F.; Maia, I. A.; J. Nanostruct. Polym. Nanocomp. 2011, 7, 47.

16. de Campos, B. M.; Bandeira, L. C.; Calefi, P. S.; Ciuffi, K. J.; Nassar, E. J.; Silva, J. V. L.; Oliveira, M. F.; Maia, I. A.; Virtual and Physical Prototyping 2011, 6, 33. 
17. Bandeira, L. C.; de Campos, B. M.; De Faria, E. H.; Ciuffi, K. J.; Calefi, P. S.; Nassar, E. J.; Silva, J. V. L.; Oliveira, M. F.; Maia, I. A.; J. Therm. Anal. Calorim. 2009, 97, 67.

18. Zhang, L.; Li, B.; Spectrochim. Acta, Part A 2009, 74, 1060.

19. Zhang, L.; Li, B.; Su, Z.; Yue, S.; Sens. Actuators, B 2010, 143, 595

20. Yu, C.; Chen, L.; Zhang, J.; Li, J.; Liu, P.; Wang, W.; Yan, B.; Talanta 2011, 85, 1627.

21. Hu, Z. Q.; Wang, X. M.; Feng, Y. C.; Ding, L.; Lu, H. Y.; Dyes Pigm. 2011, 88, 257.

22. Leite, A.; Silva, A. M.; Cunha-Silva, L.; de Castro, B.; Gameiro, P.; Rangel, M.; Dalton Trans. 2013, 42, 6110.

23. Kar, C.; Adhikari, M. D.; Ramesh, A.; Das, G.; Inorg. Chem. 2013, 52, 743.

24. Yang, Y.; Huo, F.; Yin, C.; Chu, Y.; Chao, J.; Zhang, Y.; Zhang, J.; Li, S.; Lv, H.; Zheng, A.; Liu, D.; Sens. Actuators, B 2013, 177, 1189.

25. Rathinam, B.; Chien, C. C.; Chen, B. C.; Liu, J. H.; Tetrahedron 2013, $69,235$.

26. Jiang, X.; Meng, G.; J. Lumin. 2013, 135, 227.

27. Li, M.; Lv, H.-S.; Luo, J.-Z.; Miao, J.-Y.; Zhao, B.-X.; Sensors Actuators, B 2013, 188, 1235.

28. Luan, F.; Gu, B.; Gomes, A. S. L.; Yong, K.-T.; Wen, S.; Prasad, P. N.; Nano Today 2015, 10, 168.

29. Wiersma, D. S.; Nat. Phys. 2008, 4, 359.

30. Redding, B.; Choma, M. A.; Cao, H.; Nat. Photon. 2012, 6, 355.

31. Karl, A. T.; Zimmerman, D. L.; Journal of Statistical Planning and Inference 2021, 211, 107.

32. Lawandy, N. M.; Balachandran, R. M.; Gomes, A. S. L.; Sauvain, E.; Nature 1994, 368, 436.

33. Cao, H.; Xu, J. Y.; Seelig, E. W.; Chang, R. P. H.; Appl. Phys. Lett. 2000, 76, 2997.

34. Gottardo, S.; Sapienza, R.; García, P. D.; Blanco, A.; Wiersma, D. S.; López, C.; Nat. Photon. 2008, 2, 429.

35. Wang, Y.; Ta, V. D.; Gao, Y.; He, T. C.; Chen, R.; Mutlugun, E.; Demir, H. V.; Sun, H. D.; Adv. Mater. 2014, 26, 2954.
36. Ta, V. D.; Nguyen, T. T.; Nghiem, T. H. L.; Tran, H. N.; Le, A. T.; Dao, N. T.; Duong, P.; D.; Mai, H. H.; Opt. Commun. 2020, 475, 126207.

37. Rocha, L. A.; Caiut, J. M. A.; Messaddeq, Y.; Ribeiro, S. J. L.; Martines, M. A. U.; Freiria, J. do C.; Dexpert-Ghys, J.; Verelst, M.; Nanotechnology 2010, 21, 155603.

38. Prahas, D.; Kartika, Y.; Indraswati, N.; Ismadji, S.; Chem. Eng. J. 2008, $140,32$.

39. Santhi; T.; Prasad, A. L.; Manonmani, S.; Arabian J. Chem. 2014, 7 , 494.

40. Ahmed, K.; Rehman, F.; Pires, C. T. G. V. M. T.; Rahim, A.; Santos, A. L.; Airoldi, C.; Microporous and Mesoporouns Mater. 2016, 236, 167.

41. Perilli, T. A. G.; Sicupira, D. C.; Mansur, M. B.; Ladeira. C. Q.; HOLOS 2014, 3, 264.

42. Coronell, O.; Marinas, B. J.; Zhang, X.; and Cahill, D. G.; Environ. Sci. Technol. 2008, 42, 5260.

43. Jun, B.-M.; Cho, J.; Jang, A.; Chon, K.; Westerhoff, P.; Yoon, Y.; Rho, H.; Sep. Purif. Technol. 2020, 247, 117026.

44. Setiawan, D.; Kazaryan, A.; Martoprawiro, A. M.; Filotov, M.; Phys. Chem. Chem. Phys. 2010, 12, 11238.

45. Grimm, J. B.; Muthusamy, A. K.; Liang, Y.; Brown, T. A.; Lemon, W. C.; Patel, R.; Lavis, L. D.; Nat. Methods 2017, 14, 987.

46. Lacerda, V. L.; López-Sotelo, J. B.; Correa-Guimarães, A.; HernándezNavarro, S.; Sánches-Báscones, M.; Navas-Garcia, L. M.; MartínRamos, P.; Martín-Gil, J.; J. Environ. Manage. 2015, 155, 67.

47. Mchedlov-Petrosyan, N. O.; Kholin, Y. V.; Russian J. Appl. Chem. 2004, 77,414

48. Fujii, T.; Nishikiori, H.; Tamura, T.; Chem. Phys. Lett. 1995, 233, 424.

49. Dunsbach, R.; Schmidt, R.; J. Photochem. Photobiol., A 1995, 85, 275.

50. Bockstette, M.; Wohrle, D.; Braun, I.; Schulz-Ekloff, G.; Microporous Mesoporous Mater. 1998, 23, 83.

51. da Silva, A. A.; Flor, J.; Davolos, M. R.; Surf. Sci. 2007, 601, 1118 